\title{
Epidemiology of Human Parechovirus Type 3 Upsurge in 2 Hospitals, Freiburg, Germany, 2018
}

\section{Roland Elling, ${ }^{1}$ Sindy Böttcher, ${ }^{1}$ Florian du Bois, Alexandra Müller, Christiane Prifert, Benedikt Weissbrich, Jörg Hofmann, Klaus Korn, Anna-Maria Eis-Hübinger, Markus Hufnagel, Marcus Panning}

In 2018, a cluster of pediatric human parechovirus (HPeV) infections in 2 neighboring German hospitals was detected. Viral protein 1 sequence analysis demonstrated co-circulation of different $\mathrm{HPeV}-3$ sublineages and of $\mathrm{HPeV}-1$ and -5 strains, thereby excluding a nosocomial outbreak. Our findings underline the need for HPeV diagnostics and sequence analysis for outbreak investigations.

$\mathrm{M}$ ost human parechovirus (HPeV) infections cause mild upper respiratory tract symptoms or unspecific febrile illnesses. Severe clinical manifestations, such as meningitis/ encephalitis, myocarditis, and newborn sepsis, are caused by $\mathrm{HPeV}$ type 3 (HPeV-3) and have been described in children $<3$ months of age (1). Surveillance data show endemic circulation in several countries, such as the Netherlands (2) and the United States (3), but studies have discussed the epidemic potential of HPeV-3 in other countries, including Japan (4), Australia (5), and the United Kingdom (6). Nosocomial transmission has been documented (7).

However, in most outbreak investigations, determination of $\mathrm{HPeV}$ types was performed retrospectively $(7,8)$. We report on our investigations on a cluster of $\mathrm{HPeV}$ infections in 2 neighboring hospitals in Freiburg, Germany. We provide evidence that rapid phylogenetic analysis can assist in outbreak investigations.

\section{The Study}

During routine diagnostic testing of clinical samples from infants and young children in July 2018, we

Author affilations: Medical Center-University of Freiburg Faculty of Medicine, Freiburg, Germany (R. Elling, F. du Bois, A. Müller, M.

Hufnagel, M. Panning); Robert Koch-Institute, Berlin, Germany (S. Böttcher); University of Würzburg, Würzburg, Germany (C. Prifert, B. Weissbrich); Charité - Universitätsmedizin Berlin, Berlin

(J. Hofmann); Friedrich Alexander University Erlangen-

Nuremberg, Erlangen, Germany (K. Korn); University of Bonn

Medical Centre, Bonn, Germany (A.-M. Eis-Hübinger)

DOI: https://doi.org/10.3201/eid2507.190257 detected an increase in $\mathrm{HPeV}$ cases (Figure 1). We collected $\geq 1$ clinical specimens from most patients (Table 1). During July 9-August 25, 2018, we documented 19 cases, compared with 4 (2016) and 2 (2017) from this same time span, all using the same assays and diagnostic testing algorithm. In September 2018, only 2 patients tested $\mathrm{HPeV}$-positive; no additional cases were identified during October and November 2018. HPeV diagnostic procedures were performed upon the request of the treating physician. For the detection of $\mathrm{HPeV}$, we used commercial multiplex PCR panels: FTD respiratory pathogens 21 (Fast Track Diagnostics [FTD]; Siemens Healthineers, https://www.siemens-healthineers.com) for respiratory specimens and FTD EPA for cerebrospinal fluid (CSF), plasma, and fecal samples. Patients were hospitalized on 1 ward in hospital A and 4 wards in hospital B (Appendix Figure, http://wwwnc.cdc.gov/EID/ article/25/7/19-0257-App1.pdf). We retrieved medical data on HPeV-positive patients from the hospital-based information system. We obtained written informed consent from parents or guardians.

The age of the $2018 \mathrm{HPeV}$-positive patients ranged from 10 days to 19 months (median 1 month), with $88 \%$ of patients being $<4$ months of age (Table 1). Plasma samples $(\mathrm{n}=14)$ had a diagnostic yield of $100 \%$. The median duration of hospitalization was 4 days (range 3-23 days). The main clinical symptoms of HPeV-3 cases were fever $(\mathrm{n}=$ $21 ; 100 \%)$ and poor feeding $(n=16 ; 76 \%)$ (Table 2$)$. None of our patients required admission to a pediatric intensive care unit. All of our patients were discharged from the hospital without complications.

After we detected the first cases in July 2018, we performed molecular typing of $\mathrm{HPeV}$ by amplifying and sequencing the complete viral protein 1 (VP1) genomic region (9). Of the $25 \mathrm{HPeV}$ strains detected in Freiburg in 2018, 21 were typed as $\mathrm{HPeV}-3,2$ were assigned to $\mathrm{HPeV}-1$, and 2 to HPeV-5 (Table 1). This compares with $7 \mathrm{HPeV}-1$ and $6 \mathrm{HPeV}-3$ types in Freiburg in 2016, and $3 \mathrm{HPeV}-1,3 \mathrm{HPeV}-3$, and 5 strains not typed in 2017 (Figure 1).

For phylogenetic analyses, we included $\mathrm{HPeV}$ strains detected during January 2016-September 2018 at another 4 university hospitals: Würzburg $(\mathrm{n}=56)$

${ }^{1}$ These authors contributed equally to this article. 


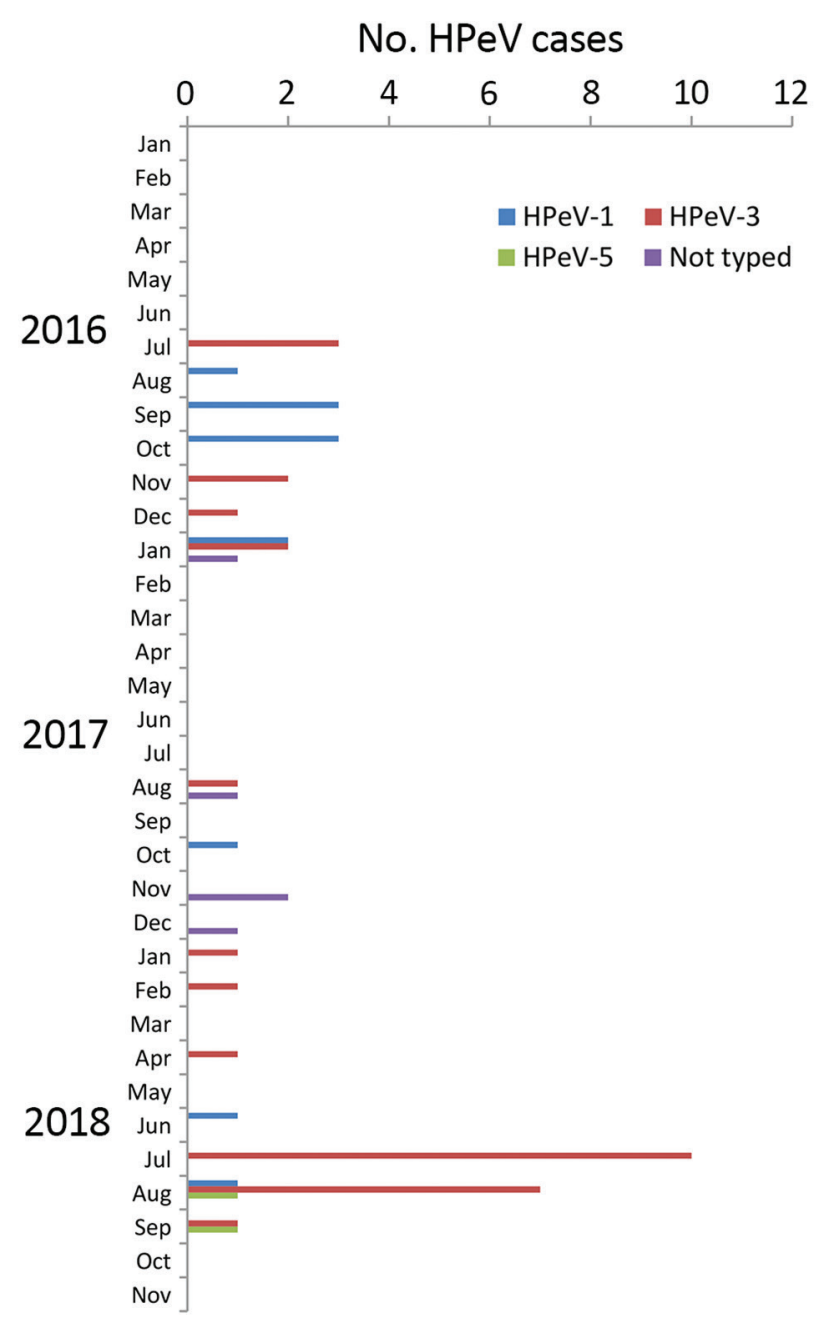

Figure 1. Number of human parechovirus (HPeV) cases in infants and young children by month, Freiburg, Germany, 2016-2018.

and Erlangen $(\mathrm{n}=10)$ in southern Germany, Bonn $(\mathrm{n}=10)$ in western Germany, and Charité Berlin $(\mathrm{n}=$ 14) in northeastern Germany. We detected $134 \mathrm{HPeV}$ strains in respiratory, fecal, CSF, and serum samples. These were typed based on the VP1 genomic region (9). We detected HPeV types 1, 3, 4, 5, and 6. We deposited all sequences in GenBank under accession numbers MK204942-MK204985 and MK291273-MK291362 (Appendix Tables 1, 2). For HPeV-3 phylogenetic analysis, we included 74 strains identified during 2016 2018 and compared them with representative reference strains available from GenBank (Figure 2). Because of high nucleotide variability in the $3^{\prime}$ end of the VP1 coding region, we included only complete VP1 sequences.

As recently described, $2 \mathrm{HPeV}-3$ lineages have been identified (10). Widespread clustering proved co-circulation of the 2016-2018 HPeV strains from Germany (Figure 2). One cluster comprising German strains was most closely related to HPeV-3 identified in Japan (98.82\% nt identity), Australia (98.82\%), and the United Kingdom (99.12\%).

Among the 21 Freiburg $2018 \mathrm{HPeV}-3$ strains, 3 groups of completely identical VP1 sequences $(10,3$, and 2 sequences) were observed. However, no separate clustering could be detected among these strains because HPeV sequences from other regions in Germany also were assigned to these groups. A direct epidemiologic link could be drawn between 2 cases (cases 12 and 14, with completely identical VP1 sequences, were in twins; Figure 2). Another 2 cases (cases 15 and 18) shared time on the same ward and also displayed $100 \%$ identical sequences. However, no hospital ward-specific clustering was observed, suggesting community-acquired transmission.

\section{Conclusions}

Routine diagnostics showed an unexpectedly high number of $\mathrm{HPeV}$ cases during a 6-week period in 2 neighboring hospitals in Freiburg, Germany. This raised concern about the possibility of a nosocomial outbreak. Recently, healthcare-associated transmission of HPeV-3 has been described. This makes timely identification of outbreaks essential from a hospital hygiene, as well as a public health, perspective (7).

Several patients showed signs of sepsis-like illness, including the clinical triad of fever, poor feeding, and irritability. This is similar to a UK case series reporting a cluster of HPeV infections among infants in 2016 (6). In our study, $\mathrm{HPeV}-3$ was detected exclusively in CSF samples, indicating a more severe clinical phenotype compared with HPeV-1 and -5 infections, supporting previous data (11). Studies have shown that rapid detection of $\mathrm{HPeV}$ reduced length of hospital stay and antimicrobial drug use. This emphasizes the usefulness of $\mathrm{HPeV}$ diagnostics (1). We showed that $\mathrm{HPeV}$ diagnostics, including molecular typing, helped to exclude a nosocomial outbreak. Diagnostically, plasma, respiratory swab, and fecal samples all showed high detection rates, and most patients were positive in $\geq 1$ area. Testing of blood samples for enterovirus detection was recently proposed for infants and should be considered for HPeV accordingly (12).

We demonstrated different $\mathrm{HPeV}$ types and sublineages, including 2 rare $\mathrm{HPeV}-5$ infections. By conducting phylogenetic analysis in combination with reviewing epidemiologic data, we could exclude a nosocomial outbreak. However, based on this information, transmissions could not be ruled out in 2 independent events with 2 cases each. Although a cluster of HPeV-3 infections has been described (6), retrospective sequence analysis showed different clustering of the identified strains (13). Because of low nucleotide variability, sequencebased differentiation between $\mathrm{HPeV}-3$ strains remains 
Table 1. Epidemiologic data for HPeV cases in Freiburg, Germany, January-September 2018*

\begin{tabular}{|c|c|c|c|c|c|c|}
\hline \multirow[b]{2}{*}{ Case no. } & \multirow{2}{*}{$\begin{array}{c}\text { Patient age, } \\
\text { mo/sex }\end{array}$} & \multicolumn{4}{|c|}{ Specimen type } & \multirow[b]{2}{*}{ HPeV type } \\
\hline & & Cerebrospinal fluid & Upper respiratory tract & Plasma & Feces & \\
\hline 1 & $2 / \mathrm{M}$ & Negative & Positive & NA & Negative & 3 \\
\hline 2 & $3 / \mathrm{M}$ & Positive & Positive & Positive & Positive & 3 \\
\hline 3 & $0 / F$ & Positive & Positive & NA & NA & 3 \\
\hline 4 & 19/M & NA & NA & NA & Positive & 1 \\
\hline 5 & $1 / \mathrm{M}$ & Positive & Positive & Positive & Positive & 3 \\
\hline 6 & $1 / \mathrm{M}$ & NA & NA & Positive & Positive & 3 \\
\hline 7 & $1 / F$ & NA & NA & NA & Positive & 3 \\
\hline 8 & $7 / \mathrm{M}$ & Negative & NA & NA & Positive & 3 \\
\hline 9 & $2 / \mathrm{M}$ & Negative & NA & NA & Positive & 3 \\
\hline 10 & $2 / \mathrm{M}$ & NA & NA & Positive & NA & 3 \\
\hline 11 & $1 / \mathrm{M}$ & NA & Positive & NA & Positive & 3 \\
\hline 12 & $0 / \mathrm{M}$ & Positive & Positive & Positive & Positive & 3 \\
\hline 13 & $1 / \mathrm{M}$ & Positive & Positive & NA & NA & 3 \\
\hline 14 & $0 / F$ & Positive & Positive & Positive & Positive & 3 \\
\hline 15 & $0 / F$ & NA & NA & Positive & Positive & 3 \\
\hline 16 & $2 / \mathrm{M}$ & NA & NA & Positive & Positive & 3 \\
\hline 17 & $2 / \mathrm{M}$ & NA & NA & Positive & Positive & 3 \\
\hline 18 & $1 / F$ & NA & NA & Positive & Positive & 3 \\
\hline 19 & $17 / F$ & NA & Positive & NA & NA & 1 \\
\hline 20 & $1 / F$ & NA & NA & Positive & Positive & 3 \\
\hline 21 & $4 / F$ & NA & Positive & NA & Positive & 3 \\
\hline 22 & $2 / \mathrm{M}$ & Positive & NA & NA & Positive & 3 \\
\hline 23 & O/M & NA & NA & Positive & Positive & 5 \\
\hline 24 & $0 / \mathrm{M}$ & Positive & NA & NA & Positive & 3 \\
\hline 25 & 1/M & NA & NA & Positive & Positive & 5 \\
\hline
\end{tabular}

Table 2. Clinical signs and symptoms of HPeV cases in Freiburg, Germany, January-September 2018*

\begin{tabular}{lccc}
\hline & & No. (\%) positive patients & \\
\cline { 2 - 4 } Clinical signs and symptoms & $\mathrm{HPeV}-1, \mathrm{n}=2$ & $\mathrm{HPeV}-3, \mathrm{n}=21$ & $\mathrm{HPeV}-5, \mathrm{n}=2$ \\
\hline Fever & $1(50)$ & $16(100)$ & $2(100)$ \\
Poor feeding & $1(50)$ & $13(62)$ & $1(50)$ \\
Irritability & 0 & $6(29)$ & $1(50)$ \\
Rash & $1(50)$ & $5(24)$ & $1(50)$ \\
Diarrhea & $1(50)$ & $5(24)$ & 0 \\
Respiratory distress & 0 & 0 & 0 \\
Vomiting & $1(50)$ & & 0 \\
\hline${ }^{*} \mathrm{HPeV}$, human parechovirus & & & 0 \\
\hline
\end{tabular}

ambiguous, a circumstance that impedes molecular outbreak investigation (14).

Our study has limitations. There is a lack of available sequence data from pediatric patients in Germany. In contrast to reports from the Netherlands and the UK, a biannual cycle of $\mathrm{HPeV}$ infections has not been demonstrated in Germany; however, our data suggest a biannual cycle. From a public health perspective, a central repository for $\mathrm{HPeV}$ sequences, together with key anonymized clinical data from human cases, would improve our understanding of $\mathrm{HPeV}$ epidemiology and virus evolution. Institutionalized surveillance similar to the enterovirus surveillance and typing systems already in place across Europe could serve as a blueprint $(8,15)$.

Our report underscores the usefulness of $\mathrm{HPeV}$ diagnostics in infants. It illustrates the power of VP1 sequenceguided phylogenetic HPeV analysis, which helped, in combination with epidemiologic data, to rapidly investigate an HPeV outbreak.

\section{About the Authors}

Dr. Elling is a pediatric infectious diseases specialist at the Centers for Pediatrics and Adolescent Medicine and Chronic Immunodeficiency in Freiburg, Germany. His research interests are pediatric infectious diseases and innate immunity. He is supported by the Berta-Ottenstein Clinician Scientist program of the Medical Faculty, University of Freiburg. Dr. Böttcher is a virologist at the German National Reference Center for Poliomyelitis and Enteroviruses at the Robert Koch-Institute in Berlin, Germany. Her research focuses on molecular virology and epidemiology of enteroviruses and parechoviruses.

\section{References}

1. Kadambari S, Harvala H, Simmonds P, Pollard AJ, Sadarangani M. Strategies to improve detection and management of human parechovirus infection in young infants. Lancet Infect Dis. 2019;19:e51-8. http://dx.doi.org/10.1016/S1473-3099(18)30288-3

2. van der Sanden S, de Bruin E, Vennema H, Swanink C, Koopmans M, van der Avoort H. Prevalence of human 


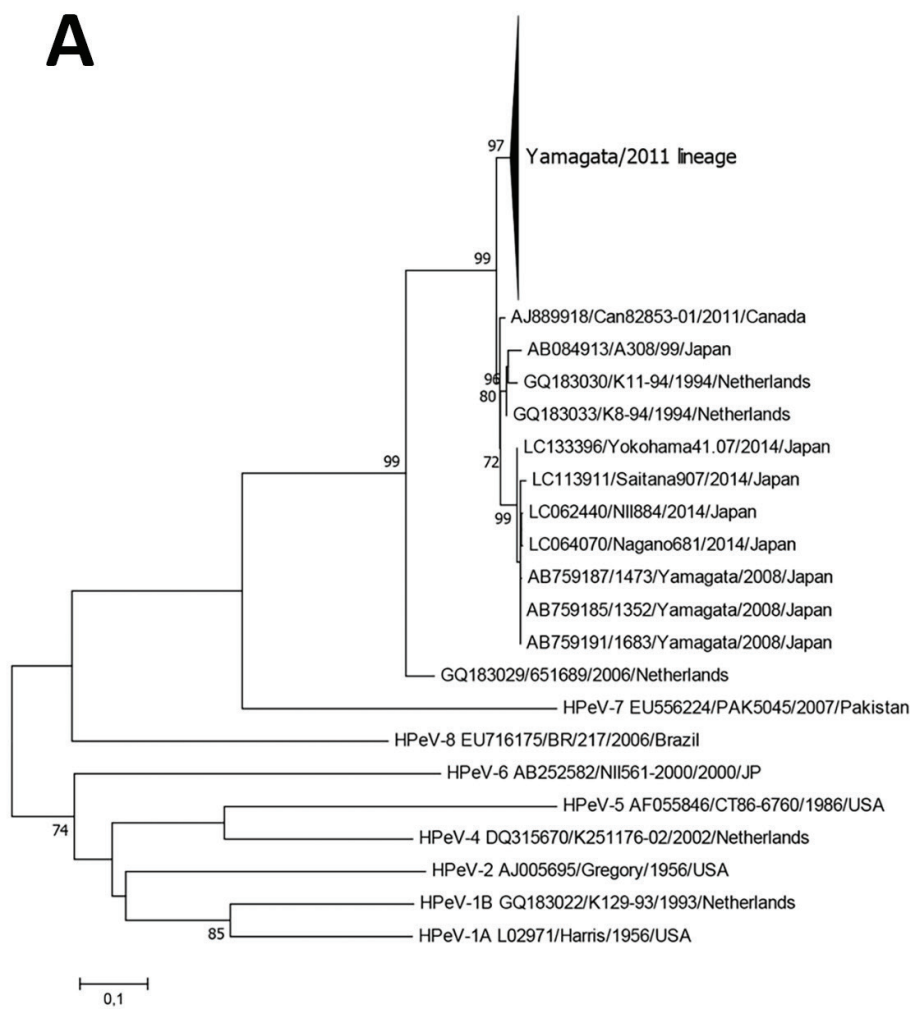

Figure 2. A) Phylogenetic analysis of human parechovirus type 3 strains collected during 2016-2018 from patients hospitalized in Freiburg, Germany, along with strains from 4 geographic regions in Germany based on the viral protein 1 region (678 nt) of the polyprotein gene $(n=74)$. B) Phylogenetic analysis of Yamagata/2011 parechovirus lineage. Color code depicts wards in the 2 Freiburg hospitals: green, A3; red, B1; pink, B3; purple, B4. Cases in twins are marked with open circles. Scale bars indicate nucleotide substitutions per site.

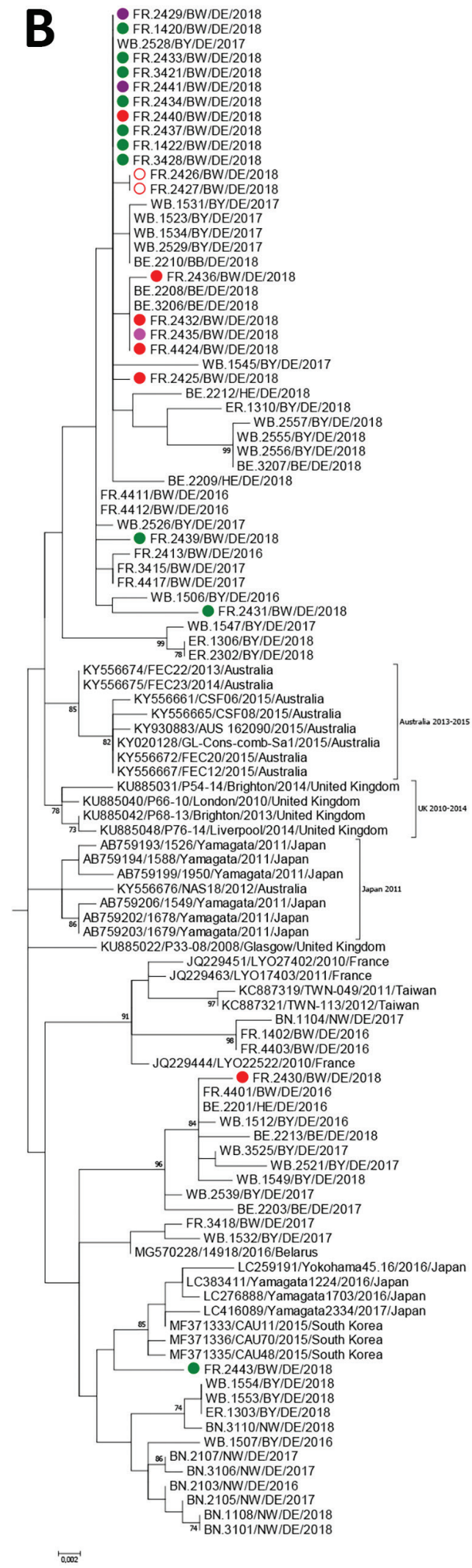


parechovirus in the Netherlands in 2000 to 2007. J Clin Microbiol 2008;46:2884-9. http://dx.doi.org/10.1128/JCM.00168-08

3. Abedi GR, Watson JT, Pham H, Nix WA, Oberste MS, Gerber SI. Enterovirus and human parechovirus surveillance-United States, 2009-2013. MMWR Morb Mortal Wkly Rep. 2015;64:940-3. http://dx.doi.org/10.15585/mmwr.mm6434a3

4. Aizawa Y, Suzuki Y, Watanabe K, Oishi T, Saitoh A. Clinical utility of serum samples for human parechovirus type 3 infection in neonates and young infants: the 2014 epidemic in Japan. J Infect. 2016;72:223-32. http://dx.doi.org/10.1016/ j.jinf.2015.10.010

5. Cumming G, Khatami A, McMullan BJ, Musto J, Leung K, Nguyen O, et al. Parechovirus genotype 3 outbreak among Infants, New South Wales, Australia, 2013-2014. Emerg Infect Dis. 2015;21:1144-52. http://dx.doi.org/10.3201/eid2107.141149

6. Tang JW, Holmes CW, Elsanousi FA, Patel A, Adam F, Speight R, et al. Cluster of human parechovirus infections as the predominant cause of sepsis in neonates and infants, Leicester, United Kingdom, 8 May to 2 August 2016. Euro Surveill. 2016;21:30326. http://dx.doi.org/10.2807/1560-7917.ES.2016.21.34.30326

7. Strenger V, Diedrich S, Boettcher S, Richter S, Maritschnegg P, Gangl D, et al. Nosocomial outbreak of parechovirus 3 infection among newborns, Austria, 2014. Emerg Infect Dis. 2016;22: 1631-4. http://dx.doi.org/10.3201/eid2209.151497

8. Ferreras Antolín L, Kadambari S, Braccio S, Tang JW, Xerry J, Allen DJ, et al.; Parechovirus Surveillance Network. Increased detection of human parechovirus infection in infants in England during 2016: epidemiology and clinical characteristics. Arch Dis Child. 2018;103:1061-6.

9. Cristanziano VD, Böttcher S, Diedrich S, Timmen-Wego M, Knops E, Lübke N, et al. Detection and characterization of enteroviruses and parechoviruses in healthy people living in the south of Côte d'Ivoire. J Clin Virol. 2015;71:40-3. http://dx.doi.org/10.1016/j.jcv.2015.08.004
10. Nelson TM, Vuillermin P, Hodge J, Druce J, Williams DT, Jasrotia R, et al. An outbreak of severe infections among Australian infants caused by a novel recombinant strain of human parechovirus type 3. Sci Rep. 2017;7:44423. http://dx.doi.org/10.1038/srep44423

11. Harvala H, Robertson I, Chieochansin T, McWilliam Leitch EC, Templeton K, Simmonds P. Specific association of human parechovirus type 3 with sepsis and fever in young infants, as identified by direct typing of cerebrospinal fluid samples. J Infect Dis. 2009;199:1753-60. http://dx.doi.org/10.1086/599094

12. Lafolie J, Labbé A, L'Honneur AS, Madhi F, Pereira B, Decobert M, et al.; Blood Enterovirus Diagnosis Infection (BLEDI) in paediatric population study team. Assessment of blood enterovirus PCR testing in paediatric populations with fever without source, sepsis-like disease, or suspected meningitis: a prospective, multicentre, observational cohort study. Lancet Infect Dis. 2018;18:1385-96. http://dx.doi.org/10.1016/ S1473-3099(18)30479-1

13. Holmes CW, Rahman S, Allen DJ, Bandi S, Tang JW. Human parechovirus cluster in the UK, 8 May-2 August 2016-sequence analysis. J Clin Virol. 2017;93:37-9. http://dx.doi.org/10.1016/ j.jcv.2017.05.018

14. Faria NR, de Vries M, van Hemert FJ, Benschop K, van der Hoek L. Rooting human parechovirus evolution in time. BMC Evol Biol. 2009;9:164. http://dx.doi.org/10.1186/1471-2148-9-164

15. Harvala H, Jasir A, Penttinen P, Pastore Celentano L, Greco D, Broberg E. Surveillance and laboratory detection for non-polio enteroviruses in the European Union/European Economic Area, 2016. Euro Surveill. 2017;22. http://dx.doi.org/10.2807/1560-7917. ES.2017.22.45.16-00807

Address for correspondence: Marcus Panning, University of Freiburg Institute of Virology, Medical Centre, Hermann-Herder-Str. 11, 79104 Freiburg, Germany; email: marcus.panning@uniklinik-freiburg.de

\section{EID SPOTLIGHT TOPIC}

Antibiotics and similar drugs, together called antimicrobial agents, have been used for the past 70 years to treat patients who have infectious diseases. Since the 1940s, these drugs have greatly reduced illness and death from infectious diseases. However, these drugs have been used so widely and for so long that the infectious organisms the antibiotics are designed to kill have adapted to them, making the drugs less effective.

Each year in the United States, at least 2 million people become infected with bacteria that are resistant to antibiotics and at least 23,000 people die each year as a direct result of these infections.

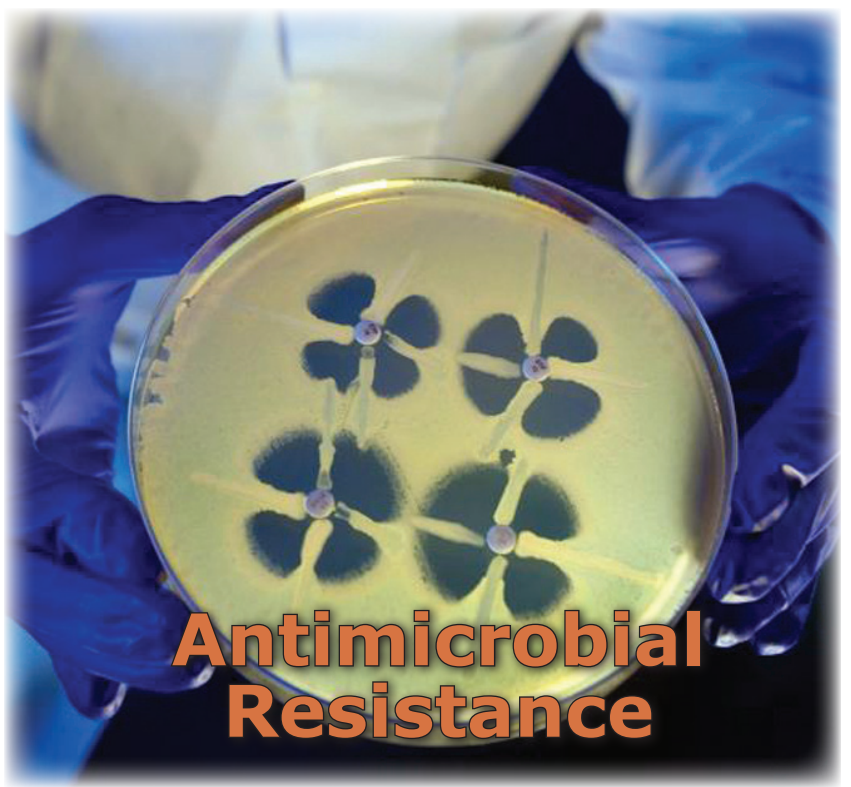

\section{EMERGING}

INFECTIOUS DISEASES http://wwwnc.cdc.gov/eid/page/resistance-spotlight 